REVIEW

\title{
Mucosal integrity and barrier function in the pathogenesis of early lesions in Crohn's disease
}

D S A Sanders

J Clin Pathol 2005;58:568-572. doi: 10.1136/icp.2004.021840

Crohn's disease aetiology is multifactorial and remains enigmatic. However, animal models show that disease heterogeneity is probable, in that more than one defective mucosal mechanism can produce the same clinical phenotype. For example, Crohn's-like lesions are reported after compromise of mucosal integrity per se in the presence of an intact immune system, through altered expression of mucosal adhesion molecules, such as cadherins and tight junction proteins, highlighting the importance of the mucosal barrier in the disease process. Key to mucosal damage is the trigger of an inflammatory cascade after luminal antigen processing, a role classically ascribed to $M$ cells in the surface follicle associated epithelium. Direct luminal antigen sampling has recently been proposed, however, by extension of dendritic cell (DC) processes through the intact gut epithelium, and it follows that early mucosal damage could result from de novo lymphoid recruitment. Cytokines, such as tumour necrosis factor $\alpha$ (TNF $\alpha$ ), are known to drive inflammation, but emerging data suggest additional important roles for TNF $\alpha$ influencing mucosal barrier efficacy by altering adhesion molecule expression, influencing epithelial apoptosis, and affecting tight junction functionality.
Correspondence to: Dr D S A Sanders, South Warwickshire General Hospitals NHS Trust, Department of Histopathology, Warwick Hospital, Lakin Road, Warwick CV34 5BW, UK: scott.sanders@swh.nhs.uk

Accepted for publication 17 December 2004
A lthough the precise aetiology of Crohn's disease remains unclear, it is thought that patients with Crohn's disease mount an abnormal immunological response to environmental antigens found within the gut lumen, with the enteric bacterial flora a strong candidate in the pathogenesis. ${ }^{1}$ Mechanisms may include exaggerated local and systemic inflammatory immune processes in combination with compromise of counter inflammatory measures.

\begin{abstract}
"It is thought that patients with Crohn's disease mount an abnormal immunological response to environmental antigens found within the gut lumen"
\end{abstract}

Antigenic ingress may be facilitated by compromise to the integrity of the mucosal lining of the bowel through the mechanisms of increased permeability, disruption, and ulceration. Once the mucosa is breached, secondary non-specific events occur-the "flood gates" are opened-the mucosa and submucosa are exposed to luminal contents, including stool and the full bacterial flora, and the morphological features of established Crohn's disease lesions become evident.

Animal models have highlighted the importance of disease heterogeneity in that more than one defect in the mucosa can lead to the same clinical disease phenotype. ${ }^{2-4}$ Just as a better understanding of the inflammatory mechanisms in Crohn's disease have expanded therapeutic options by targeting immune pathways, better understanding of the mechanisms of maintenance of the mucosal barrier, antigen sampling, and the pathogenesis of mucosal injury and repair will further facilitate the development of alternative approaches for targeting novel modalities for treatment and prevention of recurrence.

\section{EARLY CROHN'S LESIONS; A MORPHOLOGICAL PERSPECTIVE}

Morphological accounts of early Crohn's disease lesions have long suggested a link between immunity and the gut epithelium. Established bowel lesions in Crohn's disease are characterised by deep fissuring mucosal ulceration, transmural inflammation, fibromuscular hyperplasia, fibrosis, and, classically, granulomatous inflammation. These lesions represent the end stage of a complex interaction between the processes of inflammation, necrosis, healing, and repair in the gastrointestinal (GI) tract over months or even years. Observational studies of established Crohn's lesions have noted a striking relation between the vascular anatomy of the gut and the distribution of the lesions, ${ }^{5}$ and have noted chronic and granulomatous inflammation centred on the gut vasculature. ${ }^{6}$ Vascular injury is widely acknowledged to form an important feature contributing to the pathogenesis of established Crohn's lesions, but the hypothesis of a primary "granulomatous vasculitis" as the cause of Crohn's disease is not universally accepted. Rutgeerts and colleagues ${ }^{7}$ used endoscopic surveillance and biopsy of neoterminal ileum proximal to the surgical anastomosis as a model for the natural history of Crohn's disease, and observed initial early features of aphthoid ulcers, progressing to serpigenous ulcers and finally stenoses with associated ulceration after several years. Further endoscopic and histological study of early Crohn's lesions suggests that vascular lesions may be secondary to inflammatory changes, rather than the initiating event. ${ }^{8}$

Abbreviations: DC, dendritic cells; FAE, follicle associated epithelium; Gl, gastrointestinal; TNF $\alpha$, tumour necrosis factor $\alpha$ 
Conversely, there is much interest in the interaction between inflammatory cells, inflammatory cytokines, and the gut epithelium in early lesions, so that studying the early ulcerating and preulcerating lesions of Crohn's disease can provide useful insight into the pathogenesis of the disease.

Using conventional endoscopy, the earliest clinical sign of Crohn's disease is small shallow aphthoid ulcers with surrounding mucosal erythema. Specialised endoscopic techniques such as magnifying colonoscopy and fluorescent endoscopy can identify characteristic "red halo" lesions or "fluorescent spots", respectively, which histologically relate to vasodilatation, but appear to precede visible erosion. ${ }^{89}$ Most published morphological accounts of early Crohn's lesions describe mucosal lymphoid aggregates, with or without follicle formation, associated with early epithelial abnormalities, detectable initially at electron microscopy, leading to frank patchy epithelial necrosis, microerosions, and early (aphthoid) ulceration..$^{9^{10-14}}$ Neutrophilic accumulation in the surface enterocytes and crypts with epithelial degradation is also an early histological feature.

\section{INFLAMMATION AND MECHANISMS OF TISSUE INJURY}

Bacterial antigens crossing the mucosal barrier induce antigen presenting dendritic cells in the lamina propria to produce interleukin 12, which drives CD4 positive $\mathrm{T}$ cell differentiation down the $\mathrm{T}$ helper type 1 pathway. ${ }^{15}$ Inflammatory bowel disease is thought to result from inappropriate control of or an exaggerated response to the normal physiological inflammatory response. T helper type 1 cells secrete interferon $\gamma$ and tumour necrosis factor $\alpha$ $(\mathrm{TNF} \alpha)$, cytokines that trigger the biological cascade to produce tissue injury through activation of mucosal fibroblasts and upregulation of matrix degrading enzymes and growth factors. ${ }^{16}$ Novel therapeutic agents such as anti-TNF $\alpha$ (Infliximab) seek to influence the inflammatory response in Crohn's disease by targeting this pathway and by the induction of apoptosis in activated T cells. ${ }^{17}$ Recruitment of neutrophils is a key factor in early acute Crohn's lesions, mediated through upregulation of endothelial adhesion molecules and chemoattractant agents. Neutrophils play a key role in cell injury resulting from release of activated oxygen radicals (hypochlorite, superoxide, and hydroxyl radicals) and proteolytic enzymes (elastase, cathepsin G, and collagenase).

\section{FOLLICLE ASSOCIATED EPITHELIUM AND LUMINAL ANTIGEN SAMPLING}

Conventionally, adaptive immunity in the GI tract has been ascribed to specialised epithelial-like $M$ cells, found in the follicle associated epithelium (FAE) or dome epithelium overlying lymphoid tissue scattered throughout the GI tract. This lymphoid tissue is organised into Peyer's patches, discrete isolated lymphoid follicles, and lymphocyte filled villi, ${ }^{18}$ and usually comprises an organised B cell follicle, with or without a germinal centre, a mantle of $\mathrm{T}$ cells, and scattered histiocytes including dendritic cells (DC). M cells function as antigen presenting cells, regulating the sampling of luminal antigens through the mucosa and delivery to antigen presenting cells, such as macrophages and DC positioned directly below. ${ }^{19}$ DC are central to the specialised immune system in the gut, with two key functions of antigen acquisition and lymphocyte stimulation..$^{20}$ One hypothesis is that $M$ cells themselves derive from epithelial cells and are induced by particular lymphocytes in a certain microenvironment. ${ }^{21}$ Experimentally, Peyer's patch lymphocytes convert human Caco 2 cells into $M$ cells, and exposure to nonintestinal bacteria induces dramatic changes in the FAE, with increased numbers of $M$ cells. $M$ cell numbers are higher in the inflamed gut than in the normal mucosa, and $\mathrm{M}$ cell bursting at the tip of lymphoid aggregates has been noted and proposed as a mechanism of aphthoid ulceration. ${ }^{22}$ However, the absence of lymphocytes does not prevent the formation of $M$ cells experimentally, suggesting that the signalling molecules promoting $\mathrm{M}$ cell differentiation may also be supplied by non-B, non-T cells. ${ }^{23}$ Nevertheless, lympho-epithelial crosstalk plays a central role in altering the phenotype of mucosal cell populations in the GI tract.

\section{"Dendritic cells are central to the specialised immune system in the gut, with two key functions of antigen acquisition and lymphocyte stimulation"}

Traditionally, it has been suggested that aphthoid ulceration in Crohn's disease originates in the FAE, over the organised lymphoid follicles, ${ }^{9}$ which has always been thought to be the main portal of entry for potential pathogens. However, a fascinating recent paper has suggested an $M$ cell independent mechanism in rats executed by DC directly sampling luminal antigen by "unzipping" tight junctions and extending dendrites through the intact epithelium, ${ }^{24}$ an observation that has not yet been reproduced in humans. These DC express adhesion molecules, including tight junction proteins such as occludin, claudin 1 , and zonula occludens 1, and adherens junction proteins E-cadherins and $\beta$ catenin, to ensure preservation of mucosal integrity. Specific molecules involved in lymphocyte homing to the gut can be induced by gut DC, and hence DC can effectively target effector cells to the site of direct antigen sampling or challenge. In mouse models of colitis, pathogenic $\mathrm{T}$ cells clustered and proliferated in DC aggregates under the basal crypt epithelium five to 10 days before overt colitis could be detected..$^{25}$ Biopsies of the intact mucosa in patients with early Crohn's disease often contain less well organised lymphoid aggregates, lacking germinal centres and comprising a large complement of $\mathrm{T}$ cells. ${ }^{7}$ An exaggerated local immune response to antigen sampling by DC in the intact mucosa in humans with ad hoc recruitment of lymphocytes would provide one alternative hypothetical mechanism in the pathogenesis of early Crohn's lesions.

\section{MUCOSAL INTEGRITY, PERMEABILITY, AND ADHESION}

The lining of the GI tract is remarkably resilient, able to withstand a variety of noxious stimuli and rapidly responding to mucosal injury through cellular proliferation, migration, and remodelling. Peptides such as transforming growth factor $\alpha$, pancreatic secretory trypsin inhibitor, epidermal growth factor, and spasmolytic polypeptide have been proposed as mucosal guardians, with roles in mucosal integrity, luminal surveillance, and rapid response to injury. ${ }^{26}$ Epidermal growth factor enemas have been used successfully to treat acute left sided ulcerative colitis, putatively acting through several mechanisms including stimulation of cell migration and proliferation, which help to reconstitute the epithelial barrier. ${ }^{27}$ Trefoil peptides, found in the goblet cells of the intestine, promote cell migration and interact with mucins, such as MUC2, suggesting cooperativity between the two in epithelial protection. ${ }^{28}$

Epithelial barrier function is primarily regulated by tight junctions, the formation and stabilisation of which are intimately related to adherens junction assembly. ${ }^{29-31}$ For some time it has been known that increased intestinal permeability is found in patients with Crohn's disease and their unaffected family members, and it is thought that permeability is related to tight junctional alterations. Recent animal data suggest that the increased mucosal permeability 
seen in experimental colitis is secondary to intestinal inflammation, ${ }^{32}$ but the basis for changes in paracellular permeability is unclear, and no ultrastructural changes could be demonstrated in tight junctions. However, the tight junction protein occludin is reduced in inflammatory bowel disease in regions of actively migrating neutrophils and in quiescent areas of biopsies, suggesting a role in enhanced paracellular permeability. ${ }^{33}$ Conversely, enhanced epithelial integrity has been seen in response to luminal bacteria through translocation of zonula occludens 1 by the protein kinase C/Toll-like receptor 2 pathway. ${ }^{34}$

Increasingly, a link between increased gut permeability in Crohn's disease and the inflammatory cytokine TNF $\alpha$ has been noted. A role for TNF $\alpha$ in both apoptosis independent disruption of epithelial barrier function via tight junctions ${ }^{35}$ and upregulation of epithelial apoptosis (reversible by anti$\mathrm{TNF} \alpha$ antibody treatment) ${ }^{36}$ in the absence of changes in the expression of tight junction proteins is reported, suggesting that TNF $\alpha$ may be the major link between the leaky bowel and Crohn's disease. ${ }^{37}$

E-cadherin is one of the most important cell-cell adhesion molecules involved in the maintenance of integrity in the gut epithelium; it is found in the adherens junction and also extrajunctionally, forming intercellular cement on the lateral cell membrane of enterocytes. One of the most important observations made in 1995 was that chimaeric mice expressing two lineages of gut cells, one wild type and one a dominant negative with $\mathrm{N}$-cadherin substituted for $\mathrm{E}$ cadherin, developed patchy transmural inflammation in areas of leaky epithelium, characterised by $\mathrm{N}$-cadherin expression. ${ }^{38}$ Hence, a defect in gut barrier function and increased permeability can lead to a Crohn's disease-like inflammatory bowel disease in the presence of an intact immune system. Preliminary investigations in humans have demonstrated perturbation of cadherins in the FAE and perifollicular crypt epithelium in isolated lymphoid aggregates in the normal colon, characterised by membranous coexpression of E-cadherin and upregulated de novo $\mathrm{P}$-cadherin, in association with increased epithelial proliferation. ${ }^{39}$

The relative roles of $\mathrm{P}$-cadherin and E-cadherin are uncertain. Clues to the functionality of P-cadherin come from previous reported associations with mucosal proliferation, injury, ulceration, repair, and neoplastic change in the GI tract. ${ }^{40-42}$ Essentially, coexpression of P-cadherin and Ecadherin is a reversible feature of cells undergoing proliferation and migration and, although $\mathrm{P}$-cadherin upregulation may facilitate mucosal regeneration without much compromise to mucosal integrity, the probable result will be increased permeability. P-cadherin has been shown to be functional with regard to catenin binding across the cell membrane ${ }^{42}$ but there may be altered binding affinity with $\beta$ catenin, with the potential for release of $\beta$ catenin into the cytoplasmic pool, translocation to the nucleus, and potential for cell signalling and cellular proliferation. Regulation of cadherin expression in inflammation is poorly understood and there are conflicting reports of E-cadherin upregulation and conversely downregulation associated with inflammation in the gut. ${ }^{40}$ Epigenetic changes, such as downregulation of intracellular transmitter Smad4, have been associated with E-cadherin and P-cadherin downregulation ${ }^{44}$; re-expression of Smad4 induced the expression of E-cadherin and P-cadherin, with suppressed growth in tumours. ${ }^{45}$ Interestingly, cytokines, in particular TNF $\alpha$, have been shown to downregulate E-cadherin in Caco 2 cells (model of small intestine) ${ }^{46}$ and upregulate P-cadherin in prostate cells. ${ }^{47}$ Enterocytes adjacent to foci of ulceration in inflammatory bowel disease upregulate P-cadherin, ${ }^{40}$ and colonic biopsies taken from early aphthoid ulcers and from the intact mucosa between aphthoid ulcers in patients with Crohn's disease contain lympho-epithelial aggregates, ${ }^{7}$ with significantly greater epithelial P-cadherin upregulation in perifollicular crypts and the FAE compared with control follicles, associated with crypt proliferation. ${ }^{39}$

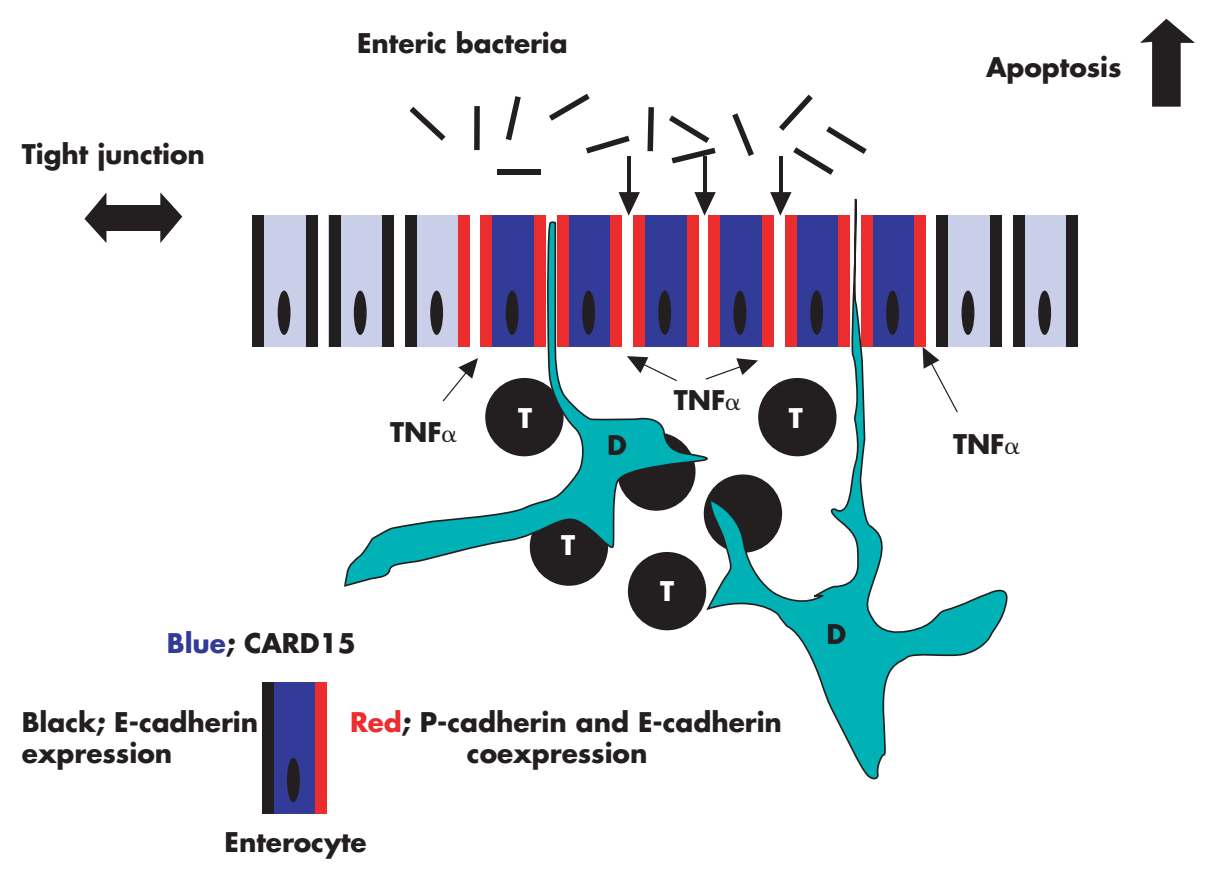

Figure 1 Putative alternative mechanisms of antigen sampling and alteration of surface cadherin expression in the gastrointestinal tract; extension of dendritic (D) cell processes between enterocytes directly samples luminal antigen with resultant lymphocyte (T; T cell) recruitment, inflammation, and cytokine production. Alteration of normal intercellular enterocyte cadherin profile (denoted in black) around lymphoid aggregates characterised by de novo P-cadherin coexpression with E-cadherin (denoted in red) under the influence of tumour necrosis factor $\alpha$ (TNF $\alpha$ ). Upregulation of cytoplasmic CARD15 in blue. The resultant increased luminal antigen ingress is denoted by bold arrows. Other suggested roles for TNF $\alpha$ include an increase in epithelial apoptosis (black arrow) and making tight junctions more "leaky" (double headed arrow) 
Hence, as in the mouse colitis model, alteration of classic cadherin expression can be demonstrated in the human gut, is a feature of the normal and inflamed gut, both precedes and results from inflammation, and may influence mucosal permeability and integrity. Whether resulting from altered adhesion or neutrophilic infiltration of the surface enterocytes with epithelial degradation, compromised mucosal integrity and increased permeability would allow underlying tissue DC more direct access to luminal antigen than formal antigen sampling through $\mathrm{M}$ cells and the FAE.

Figure 1 illustrates a hypothesis for an alternative mechanisms of antigen sampling and cytokine induced changes in barrier function; this hypothesis incorporates published animal experimental data on DC, and CARD15 and cadherin protein expression in Crohn's disease in humans.

\section{CARD 15/NOD2 GENE MUTATION}

Genetic studies have identified candidate genes that can account for the apparent genetic susceptibility to inappropriate inflammation arising from abnormal immunity to luminal antigens in Crohn's disease. Mutation of the immunosusceptibility gene CARD15/NOD 2 (caspase associated recruitment domain/nucleotide binding oligomerisation domain 2) at $16 q 12$ is the first primary defect to be identified and provides a major tool for understanding the disease processes. ${ }^{48}{ }^{49}$ CARD15 is a putative receptor of bacterial peptidoglycan, is mainly expressed within the cytoplasmic membrane of cells bridging innate and adaptive immunity, such as macrophages and dendritic cells, ${ }^{50}$ and is reported to be linked to granuloma formation. It is thought to be involved in protein-protein signalling and apoptosis, and is involved in the host response to bacterial components that induce inflammation via nuclear factor $\kappa \mathrm{B}$, the key transcription factor in immunoinflammatory responses. However, enterocyte cell lines can also upregulate CARD15 when stimulated, and CARD15/NOD2 may function as an antibacterial factor, preventing invasion by Salmonella typhimurium experimentally. ${ }^{51}$ Expression in epithelial cells may be upregulated by $\mathrm{TNF} \alpha^{52}$

\section{"A lack of inducible $\beta$ defensins has been reported in Crohn's disease, resulting in a defect in barrier function predisposing to bacterial invasion"}

In work that casts new light on the role of the Paneth cell, Ogura and colleagues ${ }^{53}$ have recently reported CARD15/NOD2 expression localised to Paneth cells located close to stem cells in crypts predominantly in the small intestine. Paneth cells are secretory cells expressing antimicrobial peptides including defensins-small cationic arginine rich peptides with a molecular weight of 3-5 kDa. The $\alpha$ defensins have a broad spectrum of antimicrobial activity and may also act as chemokines. ${ }^{54}{ }^{55}$ A link between reduced $\alpha$ defensin expression in Paneth cells in Crohn's disease and NOD2 mutations has been suggested. ${ }^{56}$ The $\beta$ defensins are expressed by epithelial cells in the GI tract and are an important factor in innate mucosal immunity. A lack of inducible $\beta$ defensins has been reported in Crohn's disease, ${ }^{57}$ resulting in a defect in barrier function predisposing to bacterial invasion.

Thus, a link between CARD15/NOD2 genotype, nuclear factor $\kappa \mathrm{B}$, and Paneth cell secretion provides one explanation for the reported association of gene mutation with ileal disease, and truncation and functional changes in the NOD2 protein may form another part of the pathophysiology of mucosal barrier disruption. However, conflicting reports are emerging on the localisation of NOD2/CARD15 in the colon, highlighting potential differences in the pathogenesis of ileal and colonic disease. ${ }^{50}$ 51 Interestingly, there is now a report of
Take home messages

- Animal models have shown disease heterogeneity in the development of Crohn's disease, with more than one defective mucosal mechanism leading to the same clinical phenotype

- Crohn's-like lesions are reported after compromise of mucosal integrity per se with an intact immune system

- Direct luminal antigen sampling by dendritic cells has been proposed as a novel mechanism of antigen processing

- New additional roles for tumour necrosis factor $\alpha$ have been proposed, including influencing mucosal barrier function through expression of adhesion molecules, apoptosis, and tight junction functionality

- Reported differences in NOD2/CARD15 expression in the gut highlight potential differences in the pathogenesis of ileal and colonic disease

CARD15 upregulation in the colonic epithelium surrounding lymphoid follicles in actively inflamed Crohn's lesions, ${ }^{58}$ acting perhaps as an intracytoplasmic receptor for bacterial products, and with staining intensity related to the severity of inflammation. This implies a more prominent role for mucosal barrier function in the pathogenesis of colonic, as opposed to small bowel, Crohn's disease.

\section{CONCLUSION}

The pathogenesis of Crohn's disease is complex and involves defects in immunoregulation and maintenance of the mucosal barrier. There is evidence to suggest that the relative contribution of each to the pathogenesis of the disease may differ between different sites in the GI tract. Defects in mucosal integrity and repair remain key elements in initiation of the disease, and a better understanding of the molecular mechanisms involved in the maintenance of the barrier function-such as antigen sampling, lympho-epithelial crosstalk, the role of the cytokine TNF- $\alpha$, and the role of epithelial CARD15/NOD2 expression-could help unravel the pathogenetic mechanisms of this fascinating disease. This approach offers great potential for the development of a more extensive range of targeted treatments in Crohn's disease.

\section{ACKNOWLEDGEMENTS}

I would like to thank Professor K Geboes for sending examples of early Crohn's lesions for study and Professor J Jankowski for encouragement and help with the manuscript.

Preliminary data on cadherin expression in early Crohn's lesions have been presented in poster and abstract form; GUT 2002;48 suppl 1 A82

\section{REFERENCES}

1 Shanahan F. Crohn's disease. Lancet 2002;359:62-9.

2 Blumberg RS, Saubermann $\amalg$, Strober W. Animal models of mucosal inflammation and their relation to human inflammatory bowel disease. Curr Opin Immunol 1999; 11:648-56.

3 Wirtz S, Neurath MF. Animal models of intestinal inflammation: new insights into the molecular pathogenesis and immunotherapy of inflammatory bowel disease. Int J Colorectal Dis 2000;15:144-60.

4 Kosiewicz MM, Nast CC, Krishnan A, et al. Th 1-type responses mediate spontaneous ileitis in a novel murine model of Crohn's disease. J Clin Invest 2001; 107:695-702.

5 Anthony A, Dhillon P, Pounder RE, et al. Ulceration of the ileum in Crohn's disease: correlation with vascular anatomy. J Clin Pathol 1997;50:1013-17.

6 Wakefield AJ, Sankey EA, Dhillon P, et al. Granulomatous vasculitis in Crohn's disease. Gastroenterology 1991;100:1279-87.

7 Rutgeerts P, Geboes K, Vantrappen V, et al. Natural history of recurrent Crohn's disease at the ileocolonic anastomosis after surgery. Gut 1984;25:665-72 
8 Maunoury V, Mordon S, Geboes K, et al. Early vascular changes in Crohn's disease: an endoscopic fluorescence study. Endoscopy 2000;32:700-5.

9 Fujimura Y, Kamoi R, lida M. Pathogenesis of aphthoid ulcers in Crohn's disease: correlative findings by magnifying colonoscopy, electron microscopy and immunohistochemistry. Gut 1996:38:724-32.

10 McGovern VJ, Goulstom SJM. Crohn's disease of the colon. Gut 1968;9:164-76.

11 Rickert RR, Carter HW. The "early" ulcerative lesion of Crohn's disease: correlative light and scanning electron microscopy studies. J Clin Gastroenterol 1980:2:11-19.

12 Dvorak AM, Connell AB, Dickerson GR. Crohn's disease: a scanning electron microscopy study. Hum Pathol 1979;10:165-77

13 Dourmashkin RR, Davies H, Wells C, et al. Epithelial patchy necrosis in Crohn's disease. Hum Pathol 1983;14:643-8.

14 Poulsen SS, Pedersen NT, Jamum S. Microerosions in Crohn's disease in rectal biopsies. Scand J Gastroenterol 1984;19:607-12.

15 Monteleone G, MacDonald $\Pi$. Interleukin 12 and Th1 immune responses in human Peyer's patches. Trends Immunol $2001 ; 22: 244-7$

16 Mueller C. Tumour necrosis factor in mouse models of chronic intestinal inflammation. Immunology 2002;105:1-8.

17 Di Sabatino A, Ciccocioppo R, Cinque B, et al. Defective mucosal T cell death is sustainably reverted by Infliximab in a caspase dependent pathway in Crohn's disease. Gut 2004;53:70-1.

18 Moghaddami M, Cummins A, Mayrhofer G. Lymphocyte filled villi: comparison with other lymphoid aggregations in the mucosa of the human intestine. Gastroenterology 1998;115:1414-25.

19 Nicoletti C. Unsolved mysteries of the intestinal M cells. Gut 2000;47:735-9.

20 Banchereau J, Steinman RM. Dendritic cells and the control of immunity. Nature 1998:392:245-52.

21 Lugering $A$, Floer $M$, Lugering $N$, et al. Characterisation of $M$ cell formation and associated mononuclear cells during indomethacin-induced intestinal inflammation. Clin Exp Immunol 2004; 136:232-8.

22 Cuvelier CA, Quatacker J, Meilants H, et al. M-cells are damaged and increased in number in inflamed human ileal mucosa. Histopathology $1994 ; 24: 417-26$

23 Debard N, Sierro F, Browning J, et al. Effect of mature lymphocytes and lymphotoxin on the development of the follicle associated epithelium and $M$ cells in mouse Peyer's patches. Gastroenterology 2001;120:1173-82.

24 Rescigno M, Urbano M, Valasina B, et al. Dendritic cells express tight junction proteins and penetrate gut epithelial monolayers to sample bacteria. Nat Immunol $2001 ; 2: 288-90$.

25 Leithauser F, Trobonjaca Z, Moller $\mathrm{P}$, et al. Clustering of colonic lamina propria CD4(+) T cells to subepithelial dendritic cell aggregates precedes the development of colitis in a murine adaptive transfer model. Lab Invest 2001;81:1339-49.

26 Playford RJ. Peptides and mucosal integrity. Gut 1995;37:595-7.

27 Sinha A, Nightingale JMD, West K, et al. Epidermal growth factor enemas with oral mesalamine for mild to moderate left sided ulcerative colitis or proctitis. N Engl J Med 2003;349:350-7.

28 Wright, NA. Interaction of trefoil family factors with mucins: clues to their mechanism of action. Gut 2001;48:293-4.

29 Itoh M, Nagafuchi A, Moroi S, et al. Involvement of ZO-1 in cadherin-based cell adhesion through its direct binding to alpha catenin and actin filaments. J Cell Biol 1997;138:181-92.

30 Boller K, Vestweber D, Kemler R. Cell-adhesion molecule uvomorulin is localized in the intermediate junctions of adult intestinal epithelial cells. J Cell Biol 1985; 100:327-32.

31 Gumbiner B, Stevenson B, Grimaldi A. The role of the cell adhesion molecule uvomorulin in the formation and maintenance of the epithelial junctional complex. J Cell Biol 1988;107:1575-87.

32 Fries W, Mazzon E, Squarzoni S, et al. Experimental colitis increases small intestine permeability in the rat. Lab Invest 1999:79:49-57.

33 Kucharzik T, Walsh SV, Chen J, et al. Neutrophil transmigration in inflammatory bowel disease is associated with differential expression of intercellular junction proteins. Am J Pathol 2001;159:2001-9.

34 Cario E, Gerken G, Podolsky DK. Toll-like receptor 2 enhances ZO-1 associated intestinal epithelial barrier integrity via protein kinase $C$. Gastroenterology 2004; 127:224-38.
35 Bruewer $\mathrm{M}$, Luegering $\mathrm{A}$, Kucharzik $\mathrm{T}$, et al. Proinflammatory cytokines disrupt epithelial barrier function by apoptosis independent mechanisms. J Immunol 2003;171:6164-72

36 Zeissig S, Bojarski C, Buergel N, et al. Downregulation of epithelial apoptosis and barrier repair in active Crohn's disease by tumour necrosis factor $\alpha$ antibody treatment. Gut 2004;53:1295-302.

37 Gibson PR. Increased gut permeability in Crohn's disease: is TNF $\alpha$ the link? Gut 2004;53:1724-5

38 Hermiston ML, Gordon Jl. Inflammatory bowel disease and adenomas in mice expressing a dominant negative $\mathrm{N}$-cadherin. Science 1995;270:1203-7.

39 Sanders DSA, Hejmadi RK, Ransford R, et al. Perturbation of classical cadherins in the lymphoid associated epithelium; further insight into the pathogenesis of early Crohn's' lesions [abstract]. Gut 2001;48(suppl 1):A82.

40 Jankowski JAZ, Bedford FK, Boulton RA, et al. Alterations in classical cadherins associated with progression in ulcerative and Crohn's colitis. Lab Invest 1998;78:1155-67.

41 Sanders DSA, Perry I, Hardy R, et al. Aberrant P-cadherin expression is a feature of clonal expansion in the gastrointestinal tract associated with healing and repair. J Pathol 2000;190:526-30.

42 Hardy RG, Brown RM, Miller SJ, et al. Transient P-cadherin expression in radiation proctitis; a model of mucosal injury and repair. J Pathol 2002; 197:194-200

43 Demetter P, De Vos M, Van Damme N, et al. Focal upregulation of Ecadherin-catenin complex in inflamed bowel mucosa but reduced expression in ulcer-associated cell lineage. Am J Clin Pathol 2000;1 14:364-70.

44 Reinacher-Schick A, Baldus SE, Romdhana B, et al. Loss of Smad4 correlates with loss of the invasion suppressor E-cadherin in advanced colorectal carcinomas. J Pathol 2004;202:412-20.

45 Muller N, Reinacher-Schick A, Baldus S, et al. Smad4 induces the tumour suppressor E-cadherin and P-cadherin in normal colorectal cancer cells. Oncogene 2002;21:6049-58.

46 Perry I, Tselepis C, Hoyland J, et al. Reduced cadherin/catenin complex expression in coeliac disease can be reproduced in vitro by cytokine stimulation. Lab Invest 1999:79:1489-99.

47 Sokoloff MH, Tso CL, Kaboo R, et al. In vitro modulation of tumour progression-associated properties of hormone refractory prostate carcinoma cell lines by cytokines. Cancer 1996;77:1862-72.

48 Hugot JP, Chamaillard M, Zouali $\mathrm{H}$, et al. Association of NOD2 leucine-rich repeat variants with susceptibility to Crohn's disease. Nature 2001;411:599-603.

49 Ogura $\mathrm{Y}$, Bonen DK, Inohara N, et al. A frameshift mutation in NOD2 associated with susceptibility to Crohn's disease. Nature 2001;411:537-9.

50 Gutierez O, Pipaon C, Inohara N, et al. Induction of NOD2 in myelomonocytic and intestinal cells via nuclear factor-kappa B activation. J Biol Chem 2002;277:41701-5

51 Hisamatsu T, Suzuki M, Reinecker HC, et al. CARD15/NOD2 functions as an antibacterial factor in human intestinal epithelial cells. Gastroenterology 2003:124:993-1000.

52 Rosenstiel P, Fantini M, Brautigam K, et al. TNF-alpha and IFN-gamma regulate the expression of NOD2(CARD15) gene in human intestinal cells. Gastroenterology 2003;124:1001-9.

53 Ogura Y, Lala S, Xin W, et al. Expression of NOD2 in Paneth cells: a possible link to Crohn's ileitis. Gut 2003:52:1591-7.

54 Chertov O, Michiel DF, Xu L, et al. Identification of defensin-1, defensin-2, and CAP37/azurocidin as T-cell chemoattractant proteins released from interleukin-8-stimulated neutrophils. J Biol Chem 1996;271:2935-40.

55 Lehrer RI, Barton A, Daher KA, et al. Interaction of human defensins with Escherichia coli. Mechanism of bactericidal activity. J Clin Invest 1989;84:553-61

56 Wehkamp J, Harder, Weichenthal M, et al. NOD2 (CARD 15) mutations in Crohn's disease are associated with diminished mucosal $\alpha$-defensin expression. Gut 2004:53:1658-64.

57 Wehkamp J, Harder J, Weichenthal $M$, et al. Inducible and constitutive betadefensins are differentially expressed in Crohn's disease and ulcerative colitis. Inflamm Bowel Dis 2003;9:215-23.

58 Berrebi D, Maudinas R, Hugot J-P, et al. CARD15 gene overexpression in mononuclear and epithelial cells of the inflamed Crohn's disease colon. Gut 2003;52:840-6. 